\title{
El presupuesto participativo: un balance de su estudio y evolución en México
}

\author{
María Luisa García Bátiz, ${ }^{*}$ Luis Téllez Arana**
}

Perfiles Latinoamericanos, 26(52) | 2018

DOI: $10.18504 / \mathrm{pl} 2652-012-2018$

Recibido: 13 de octubre de 2016

Aceptado: 9 de julio de 2017

\begin{abstract}
Resumen
La creciente expansión de presupuestos participativos $(\mathrm{PP})$ registrada a partir de la década de 1980 en diferentes gobiernos locales del mundo, ha sido de gran interés para la comunidad académica internacional; no obstante, en México este tipo de experiencias no cuenta con un análisis sistemático de su desarrollo y comportamiento. Por tal razón, en este artículo se estudia la evolución del PP en México, desde la comprensión de su introducción y permanencia analizando los factores contextuales socioeconómicos e institucionales y sus dinámicas de participación que lo han propiciado, hasta una breve exposición de los retos que enfrenta y una propuesta de nuevas hipótesis de trabajo.
\end{abstract}

\begin{abstract}
The growing expansion of participatory budgeting $(\mathrm{PB})$ registered since the eighties in different local governments around the world, has been of great interest to the international academic community; nevertheless, the experiences of PB in Mexico do not have a systematic analysis of their development and behavior. Therefore, the evolution of PB in Mexico is studied. In order to understand the introduction and permanence of the $\mathrm{PB}$, some socioeconomic and institutional contextual factors and the dynamics of participation of the $\mathrm{PB}$ are examined. Finally, challenges of the experiences and working hypothesis are identified.
\end{abstract}

Palabras clave: presupuesto participativo, participación ciudadana, gobiernos locales mexicanos, democracia participativa, innovaciones democráticas, política pública local, México.

Keywords: Participatory budgeting, citizen participation, Mexican local governments, participatory democracy, democratic innovations, local public policy, Mexico.

* Doctora en Ciencias Sociales, por la Universidad de Guadalajara (México). Universidad de Guadalajara. Departamento de Estudios Regionales | maluisagarciabatiz@gmail.com

** Maestro en Políticas Públicas por la Universidad de Guadalajara (México). Universidad de Guadalajara. Doctorado en Políticas Públicas y Desarrollo | luista_1988@live.com 


\section{Introducción}

- a proliferación de nuevos mecanismos de participación ciudadana forma parte de un entorno de cambios políticos, económicos y culturales experimentados por las sociedades contemporáneas a partir de la década de 1980; principalmente, los relacionados al avance paulatino de la democracia y la descentralización. El ambiente creado por estos cambios ha sido propicio para la aparición y difusión de políticas innovadoras de participación ciudadana en los gobiernos locales; entre ellas el presupuesto participativo (PP), considerado como un mecanismo paradigmático de democracia participativa cuyo fin es involucrar a la ciudadanía en la priorización y decisión sobre el gasto público en el orden de gobierno local. El PP surgió en Brasil en 1989 y hoy en día se ha extendido a prácticamente todo el mundo gracias, en gran parte, a las atribuciones democráticas de su diseño y resultados.

Durante la diseminación global del PP se pensó que México, al contar en los años noventa y principios de los dos mil con las experiencias aisladas e inéditas del municipio de Cuquío, Jalisco y otras tres en delegaciones del Distrito Federal (D. F.) de este tipo, ${ }^{1}$ había entrado a ese auge internacional de la gestión participativa de los recursos públicos locales. No obstante, ahora luce optimista la creencia de que al tener algunas prácticas tempranas estábamos integrándonos en esa ola de la democracia participativa.

El surgimiento de estas nuevas experiencias no ha pasado desapercibido para la comunidad académica internacional y nacional, sin embargo, en México ha despertado poco interés y no existe un análisis que sistematice su avance en el país. En este marco, dos cuestionamientos guían el presente trabajo: ¿cuál ha sido la evolución del presupuesto participativo en México? y ¿qué factores nos ayudan a entender su introducción y permanencia? Lo que se pretende es evaluar en términos exploratorios el desarrollo de las experiencias del PP en México y con ese propósito se determina el surgimiento y duración de dichas experiencias indagando en las características socioeconómicas y los contextos institucionales que han influido sobre su introducción y permanencia. El propósito es lograr una primera aproximación al tema que permita derivar nuevas hipótesis de trabajo.

1 El estudio se realizó durante el periodo previo y reciente a la reforma política al Distrito Federal de 2015, por lo tanto, no se tomará en cuenta la nueva figura legal de la capital del país. De manera general, en el artículo 122 de la Constitución Política de los Estados Unidos Mexicanos la figura del Distrito Federal se sustituye con la de la Ciudad de México a la que se reconoce como entidad federativa y se da paso a la adopción de su propia constitución política, mientras que las delegaciones pasan a ser alcaldías. 
El artículo se divide en tres partes, en la primera se hace una revisión del estudio y la expansión del PP a nivel internacional y nacional, ubicando la posición de las experiencias mexicanas en este contexto, además de que se muestra la metodología del estudio; en la segunda, se identifica la extensión geográfica y temporal de las experiencias, analizando las características socioeconómicas y los contextos institucionales que las influyen y las dinámicas de participación utilizadas. Finalmente, se presentan las conclusiones derivando hipótesis de trabajo y resaltando los principales avances y retos en México en relación con el PP.

\section{El estudio de los presupuestos participativos de México}

El presupuesto participativo nació en un contexto de democratización y descentralización de los países latinoamericanos, en donde los gobiernos locales comenzaron a experimentar con mecanismos participativos para llevar una gestión pública más democrática. Surgió en el municipio de Porto Alegre, Rio Grande do Sul, Brasil ${ }^{2}$ en 1989 con la premisa de implicar a la ciudadanía en los procesos de decisión del gasto público del gobierno local. Aunque no reconocido inicialmente, su éxito fue rotundo, una vez que agrupaciones y organismos, primero nacionales y luego internacionales, dirigieron su mirada hacia dicha experiencia (Oliveira, 2011: pp. 132-141); y de ahí, a la actualidad, se ha extendido en casi todo el mundo, y no es raro que un buen número de académicos e investigadores tengan su atención sobre él.

Los primeros pasos de la expansión del PP se registran en un nivel regional en los alrededores de Porto Alegre; luego, en los años noventa, algunos gobiernos locales de Brasil y Uruguay lo implementaron; más adelante, en la misma época y ya entrado el nuevo siglo, en Latinoamérica, se dieron experiencias en Ecuador, Perú y Argentina, casi al mismo tiempo que los de Europa y Norteamérica. Finalmente se le empieza a ver en África y Oceanía (Cabannes, 2004; Sintomer, Herzberg \& Allegretti, 2010: pp. 9-10). Es difícil calcular la cantidad precisa de experiencias para cada continente pues depende de la rigurosidad de su definición (Goldfrank, 2006), pero en 2014 Montecinos (2014: p. 352) la estimó en aproximadamente tres mil, cada una con características propias.

La extensión del PP se atribuye a factores como la presencia de académicos, alcaldes, funcionarios y asesores en encuentros internacionales de prácticas

2 Hay autores que difieren sobre su origen ya que otros gobiernos municipales brasileños en los años setenta y algunos de Venezuela y Uruguay los implementaron al mismo tiempo que Porto Alegre (Goldfrank, 2006: pp. 4-5) con dinámicas bastante parecidas; no obstante, su denominación se originó en Porto Alegre, que fue donde tomó forma sistemática por diversas razones políticas y sociales. 
exitosas de gestión local; la intervención de la sociedad civil organizada local que pugna para su adopción; la influencia de organismos internacionales que los recomiendan y promueven con apoyos y financiamientos; la pluralización política, sobre todo de partidos socialdemócratas latinoamericanos, ${ }^{3}$ y la promoción formal de gobiernos nacionales y regionales (Gomes, 2011; Oliveira, 2011; Sintomer, Herzberg \& Allegretti, 2010). Hoy el PP goza de alta aceptación entre los gobiernos locales y fuerte interés entre los académicos por estudiarlo.

\section{Revisión de los estudios internacionales}

Es tal la importancia y cantidad de trabajos acerca del PP en el ámbito académico internacional que es complejo seguirlos con el debido rigor; empero, siguiendo a Montecinos (2014), los estudios internacionales se pueden ordenar agrupándolos con dos criterios: a) por etapas de acuerdo al carácter histórico de su expansión (como los antecedentes que vimos más arriba), ${ }^{4}$ y b) de acuerdo a su objeto de estudio mediante tres generaciones que coinciden con las fases de su expansión.

La primera generación son los estudios que se concentran en la etapa de experimentación y que se distinguen por mostrar las bondades o beneficios del PP por medio del análisis sobre todo de casos brasileños, los cuales destacan que la práctica de esa experiencia profundiza la democracia ${ }^{5}$ (Montecinos 2014; Goldfrank, 2006; Sousa, 2004; Wampler et al., 2006, entre otros) y fortalece el ejercicio y las acciones de gobierno (Avritzer, 2004; Goldfrank, 2012; Sintomer, 2005).

La segunda generación agrupa los estudios que describen la etapa de extensión dentro y fuera de Brasil, los cuales se preocupan por establecer las condiciones de los lugares que explican el éxito o fracaso de los PP; en esta línea, Goldfrank (2006: p. 7) sintetiza algunas variables propuestas por diferentes académicos, como la voluntad política, el capital social, las capacidades del per-

3 Recientemente, cualquier partido político lo promueve, así, este factor fue determinante al inicio; en el trabajo de Montecinos (2011) se observa que ahora el factor del partido político tiene un peso más cualitativo que cuantitativo.

4 Las etapas que propone Cabannes (2004) como experimentación y extensión en Brasil y su expansión fuera de este país.

5 Como "la representación de los excluidos y el pluralismo de la democracia (Nylen, 2003), el fortalecimiento de la rendición de cuentas (Wampler, 2004); la revalorización del ciudadano (Buenrostro, 2004) y el aporte de la superación de la pobreza, el fortalecimiento del capital social y la ciudadanía [...] (Gómez, 2007; Navarro, 1998; Baiochi, 2001)" (Montecinos, 2014: p. 355). 
sonal involucrado, el tamaño del municipio, los recursos, la plataforma legal o marcos institucionales, y la descentralización política.

Finalmente, la tercera generación son los estudios interesados en explicar las relaciones entre instituciones representativas y las participativas (Montecinos, 2011,2014) y se enfocan en una discusión política de la democracia sobre cómo las figuras representativas han terminado dominando a las participativas, por lo que no se habla de una complementariedad real entre ambas sino de que se han relacionado mediante la subordinación.

Las dos últimas generaciones de estudios han coincidido en señalar los claroscuros de las experiencias al precisar que no siempre promueven valores democráticos ni los resultados para el desempeño de los gobiernos son siempre positivos; asimismo, han encontrado que las razones de estos problemas se atribuyen a los factores condicionantes de la aparición y desarrollo del PP.

Sin embargo, una limitante de esta agrupación general de la literatura es la dificultad de identificar con claridad los casos particulares sujetos de estudio; de su revisión se puede constatar que en su mayoría no abordan el caso mexicano o se le analiza en conjunto con otros países de la región, aunque es común que solo se examinen las experiencias en el D. F. (Cabannes, 2005; Goldfrank, 2006; Sintomer, Herzberg \& Allegretti, 2010; Welp, 2016). También ha faltado más profundidad metodológica, lo que impide tanto conocer el statu quo de los PP en el país, como reconocer o explicar los problemas que los mismos enfrentan para emerger, estabilizarse y tener dinámicas de participación prometedoras. $\mathrm{Al}$ parecer, tampoco han recibido suficiente atención de los académicos internacionales más destacados.

\section{Revisión de los estudios locales}

El auge de las experiencias de participación ciudadana en México se entiende en un contexto de democratización y de reforma del Estado que, entre otros asuntos, buscaron dar a la sociedad apertura a procesos y espacios de poder de decisión. Los sucesos más trascendentes en este sentido han sido el desarrollo institucional de la democracia representativa, de los derechos humanos y la transparencia (Olvera, 2010), así como de ciertas políticas de participación ciudadana en los gobiernos locales que, en comparación con otros países de Latinoamérica, han sido insuficientes tanto en su avance como en su entendimiento (Olvera, 2010).

Las primeras experiencias formales surgen en la década de 1980 con el Sistema Nacional de Planeación y luego en los noventa con el Programa Nacional de Solidaridad, cuando se decidió incluir la ciudadanía en la planeación 
y gestión del desarrollo local (García, 2006; Olvera, 2009). En la actualidad hay experiencias del gobierno federal como consejos consultivos, comités de padres de familia, financiamientos que se dan a la sociedad civil y comités de gestión de áreas naturales (Olvera, 2009: pp. 9-12). Mientras que en los gobiernos locales se registran experiencias como los consejos vecinales, los consultivos, los de planeación, los de medio ambiente y los sociales; además de las de democracia directa; y recientemente y con menor frecuencia, las de democracia participativa como redes, consejos de responsabilidad compartida, contralorías sociales y presupuestos participativos. ${ }^{6}$

Es posible dividir los estudios de estas experiencias en tres etapas: en la primera se describen y señalan las virtudes democráticas de algunas prácticas exitosas, mostrando algunas debilidades y puntos críticos (Merino, 1994; Ziccardi \& Solano, 1995; Ziccardi, 1998); en la segunda, se agrupa y clasifica a tales experiencias para mejorar su entendimiento y precisar los niveles de participación (Canto, 2010; Díaz \& Ortiz, 2011; Gurza \& Isunza, 2010; Hevia \& Isunza, 2010; Ziccardi, 2010); y en la tercera, el objetivo es contribuir al conocimiento de las experiencias e identificar factores que inciden en los procesos de participación en contextos locales (Bolos, 2003; García, 2006, 2009; Olvera, 2009; Ziccardi, 2004).

Los estudios internacionales de la segunda y tercera etapas ya mencionadas incluyen un amplio conjunto de investigaciones sobre procesos de participación ciudadana en municipios que han explorado diversos factores que inciden en el funcionamiento de los mismos; por ejemplo, se ha examinado el papel que tienen el contexto (Schneider, 2007; Schneider \& Welp, 2011), los recursos locales (Font, Della Porta $\&$ Sintomer, 2014) y las capacidades, los marcos institucionales, y la dinámica de la participación (Montecinos, 2005).

A su vez, en varios estudios nacionales el PP es señalado unánimemente como una buena práctica que fortalece la participación ciudadana en la gestión de los recursos públicos locales; en otros, no obstante, se diluye en sus análisis junto al resto de los mecanismos estudiados, volviéndolo imperceptible y atribuyéndole indiferentemente los mismos problemas; pero en ninguno de tales estudios se ofrecen los detalles del PP, más que para Cuquío, Jalisco, ${ }^{7}$ y tampoco profundizan en los aspectos que explican diversas experiencias de participación, tal como sí sucede en los estudios internacionales citados más arriba.

6 Los problemas con las primeras experiencias nacionales y algunas locales son que han terminado creando clientelas para el partido en turno, que solo refuerzan lazos con la sociedad civil más organizada, o que han llegado a tener bajos perfiles democráticos (Díaz \& Ortiz, 2011).

7 Algunas bases de datos nacionales sobre municipios ofrecen información de prácticas participativas, pero el presupuesto participativo no se contempló en sus cuestionarios sino hasta 2015. Sin embargo, hasta el momento sus resultados no son públicos (INEGI/INDESOL, 1995, 200; INEGI, 2009). 
Por otra parte, hay trabajos acerca de los PP del país que conforman dos corrientes: la que muestra información general de experiencias concretas, y la que realiza análisis de estas desde una lente más crítica, debatiendo su surgimiento y diseños.

En la primera corriente se encuentra el conocimiento descriptivo de experiencias como la de Cuquío, Jalisco — que perduró de 1993 a 2002, y no fue reconocida inicialmente como PP aunque tuviera rasgos similares (Bazdresch, 2001; Bey, 2007; Hernández \& Velasco, 2012)—; la de Tepic, Nayarit, vigente entre 1970 y 1972 (Hernández \& Velasco, 2012); las de San Pedro Garza García de 2003 a la fecha y Santa Catarina de 1997 a 2011, en Nuevo León (Rodríguez, Annett \& Arango, 2013); la de Ecatepec de Morelos, Estado de México, de 2006 a 2009 (Ramírez, 2011), y la de los municipios de la Zona Metropolitana de Guadalajara a partir de 2011 (Briseño, 2011; Bautista, 2011; Hernández \& Velasco, 2012).

En la segunda corriente predominan los análisis de su implementación en Cuquío, Jalisco ${ }^{8}$ y de las experiencias del D. F. La de Cuquío consistió en la creación del Consejo Democrático Municipal (Codemuc) con ciudadanía representativa de consejos comunitarios conformados en sus distintas localidades que, mediante procedimientos participativos complejos, gestionaron y decidieron principalmente sobre el uso de recursos municipales para obra pública. El impulso desde la comunidad campesina y su fuerte actividad en el gobierno local llevó a que los análisis se enfocaran en cómo las organizaciones comunitarias se coordinan y colaboran con dicho gobierno local, y en el rol de las organizaciones sociales, la acción colectiva, las acciones de la burocracia y la cultura política (Bolos, 2003; Arellano, 2006; Rodríguez \& Quintana, 2003).?

Las primeras experiencias de PP en el D. F. datan de 2001 y se dieron en las delegaciones ${ }^{10}$ Tlalpan, Cuauhtémoc y Miguel Hidalgo, aunque no lograron concretarse a largo plazo y carecieron de un diseño homogéneo. Posteriormente,

8 En 2001 y por tratarse de una buena práctica recibió el Premio Gobierno y Gestión Local que otorga el CIDE (Bazdrech, 2001); esto ha beneficiado su difusión nacional.

9 La literatura destaca la influencia determinante de la comunidad campesina por medio de la Organización Campesina Independiente de Jalisco y la asesoría de las organizaciones civiles Acción Ciudadana para la Educación, la Democracia y el Desarrollo y del Instituto Mexicano para el Desarrollo de la Comunidad, así como la de algunas universidades locales y organizaciones religiosas (Bolos, 2003).

10 Previo a su reforma política, la base de organización política y división administrativa del D. F. fue la delegación que ahora pasa a ser alcaldía. Las alcaldías entran en funciones a partir de 2018, entre las diferencias con las delegaciones están que tendrán mayores facultades sobre el manejo del presupuesto, la emisión de licencias de obras y giros mercantiles, el desarrollo urbano, medio ambiente y otros servicios públicos. 
la Asamblea Legislativa del D. F. reformó la Ley de Participación Ciudadana para institucionalizar el presupuesto participativo en 2010, activo a la fecha (Marquina \& Alonzo, 2012; Olvera, 2009). Los estudios de estos casos analizan las formas de organización ciudadana inscritas en el diseño institucional (Marquina \& Alonzo, 2012), la gestión del gobierno sobre los procesos de participación ciudadana en las experiencias de los PP para la democracia local (Delgado, 2006; García, 2014) y la relación de los problemas institucionales formales (Tellez \& García, 2016).

Dichos trabajos sobre el PP permiten conocer las experiencias, pero no aspiran a sistematizarlas y tampoco discuten los factores que ayudarían a entender su introducción y permanencia, además, como sus enfoques analíticos son distintos dificultan profundizar en las condiciones del desarrollo y resultados de dicho mecanismo.

\section{Metodología de la investigación}

Como se mencionó, investigaciones previas han determinado múltiples factores que explican el funcionamiento de los procesos y presupuestos participativos, entre los más relevantes, la voluntad política, el capital social, las capacidades, el tamaño del municipio, los recursos, la plataforma legal o marcos institucionales, la dinámica de la participación, el contexto, y la descentralización política (Goldfrank, 2006: p. 7; García, 2006; Montecinos, 2005; Schneider, 2007; Schneider \& Welp, 2011; Font, Della Porta \& Sintomer, 2014).

La lista es larga y compleja por lo que ahondar en cada aspecto o ampliar el debate a nuevas explicaciones rebasa el propósito de este documento; adicionalmente, el tipo de información a la que se tuvo acceso impide dar un seguimiento a detalle de cuestiones específicas. Por ello, la propuesta de este artículo es ofrecer una primera aproximación al PP de la que deriven nuevas hipótesis de trabajo. Con ese fin se ha hecho una selección de las más importantes variables en estudios comparativos para guiar el análisis. De este modo se pondrán a la vista los factores que influyen en la introducción y permanencia de los PP identificados, se trata de los siguientes:

- El contexto socioeconómico. Del que se revisa el nivel socioeconómico de las localidades en las que se implementa.

- Los contextos institucionales. De los que se revisan las condiciones legales referentes a las escalas nacional y local, al presupuesto involucrado, al arribo de nuevos gobiernos y al papel de los actores. 
- La dinámica de participación. De la que se revisan los objetivos, alcances e intensidades de los ejercicios de participación ciudadana.

Con relación a las fuentes de investigación, se ha echado mano esencialmente de información documental obtenida por medios de comunicación, publicaciones académicas, páginas web, portales de transparencia y otras fuentes oficiales; asimismo, para la búsqueda, recuperación y corroboración de la información se utilizó una estrategia de cuatro etapas:

a) Mediante la Web, ${ }^{11}$ se realizó una búsqueda por cada estado del país con un determinado orden de palabras: "estado presupuesto participativo" (por ejemplo: Sonora presupuesto participativo).

b) Se repitió la dinámica para los municipios más poblados y los pequeños que mostraron relación.

c) En lo posible, se confrontó la información comparándola con la de las páginas web oficiales.

d) Cuando se obtenía una relación positiva, se identificaron dos criterios básicos del diseño del presupuesto participativo: contar con recursos asignados por alguna figura de gobierno local, ${ }^{12}$ y contar con un proceso de decisión participativa entre gobierno y sociedad.

De las experiencias de presupuesto participativo reconocidas se construyó una base de datos organizada según la naturaleza de estos y las variables en búsqueda. Una limitante para realizar esta investigación fue el acceso a información completa; así, conviene anticipar que gran parte de los datos que se muestran aquí corresponde a la totalidad de los casos y que cuando el acceso se presenta restringido al dato en específico ello será referenciado a pie de página.

\section{La evolución del presupuesto participativo en México}

En esta sección se exponen los resultados del estudio. En el primer apartado se ubica dónde y cuándo surgen las experiencias con PP y en los subsiguientes se revisan los factores socioeconómicos, institucionales y la dinámica de la participación ciudadana.

11 Se utilizó el buscador de Google en enero-febrero de 2015 y en abril de 2016.

12 Principalmente, que hubiera evidencia de asignación de recursos o de obras, servicios o acciones públicas implementadas por el municipio producto del mecanismo participativo. 
El avance del PP en México ha sido progresivo, especialmente de 2013 a la fecha. Y las primeras experiencias lo más parecidas a ello se dieron en $1970^{13}$ y en $1993,{ }^{14}$ aunque no se las ha reconocido como tales. Este papel se ha asignado a las desarrolladas en 1997 y a las de los años dos mil en adelante. Actualmente se registran $37 \mathrm{PP}$ promovidos por primera vez, ${ }^{15}$ con 33 experiencias aplicadas por gobiernos municipales o delegacionales y otras cuatro, con promedio de vida de 2.5 ańos, por parte de gobiernos de entidades federativas (tabla 1).

Para precisar la duración de los PP se les ha clasificado en activos e inactivos. Los activos son quince, suman el $40 \%$ del total y tienen una vida de 1.75 años; este promedio descarta los del D. F. y el de Tlajomulco de Zúniga, Jalisco, que, con seis años cada uno, son más longevos (gráfica 1). Los inactivos son 22, representan el $60 \%$ del total y han tenido un promedio de vida de 2.5 ańos. Una estimación que descarta las experiencias de Cuquío, Jalisco (la más longeva) y la de Durango, las cuales han durado nueve y seis años, respectivamente.

\section{Gráfica 1. Fechas y cantidades de la primera activación de presupuestos participativos} en México a 2016

Cantidad de experiencias por año

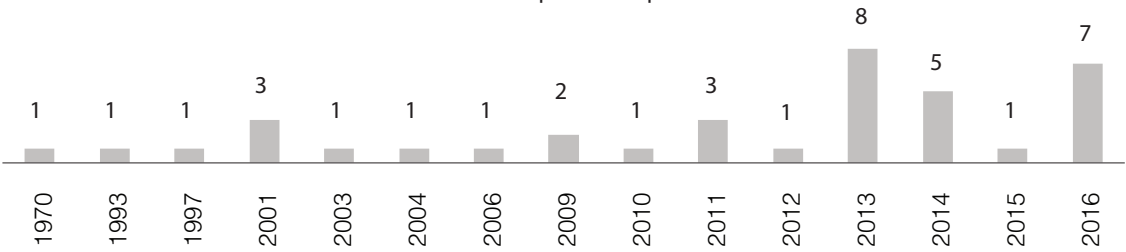

Fuente: Elaboración propia.

La totalidad de las experiencias de PP se registra en gobiernos locales de catorce entidades federativas cuya población es de treinta millones de personas que equivalen al 27\% del total del país (INEGI, 2010); debe agregarse, que los criterios de cobertura y participación son muy heterogéneos por lo que no es posible afir-

13 En Tepic, Nayarit (1970-1972), con la llegada del Partido Socialista se formaron los Comités del Pueblo, los cuales recibían una partida presupuestal cuyo destino decidían los pobladores, para luego reportarse al gobierno local (Hernández \& Velasco, 2012: pp. 275-276); de modo similar al caso de Montevideo hasta 1995.

14 En Cuquío no recibió el nombre de presupuesto participativo, aunque el proceso fue similar.

15 Destacan siete municipios en los que habían terminado y fueron reactivados ańos después. 
mar que alcancen a toda la población. En suma, menos de la mitad de las entidades federativas de la república mexicana han tenido ese tipo de participación.

Tabla 1. Oferta de presupuestos participativos en México a 2016

\begin{tabular}{|c|c|c|c|c|c|c|c|}
\hline \multirow{2}{*}{ Estado } & \multirow{2}{*}{ Municipio } & \multirow{2}{*}{$\begin{array}{l}\text { Gobierno donde } \\
\text { se implementa }\end{array}$} & \multicolumn{4}{|c|}{ Fechas de inicio y reinicio } & \multirow{2}{*}{ Estatus } \\
\hline & & & Inicio & Fin & Inicio & Fin & \\
\hline Aguascalientes & Aguascalientes & Municipal & 2012 & 2012 & - & - & Inactivo \\
\hline Chiapas & Palenque & Municipal & 2015 & 2015 & - & - & Inactivo \\
\hline Coahuila & Saltillo & Municipal & 2014 & 2014 & - & - & Inactivo \\
\hline \multirow{4}{*}{ D. F. } & En delegaciones & D. F. & 2011 & - & - & - & Activo \\
\hline & Tlalpan & Delegación & 2001 & 2003 & - & - & Inactivo \\
\hline & Miguel Hidalgo & Delegación & 2001 & 2005 & - & - & Inactivo \\
\hline & Cuauhtémoc & Delegación & 2001 & 2001 & - & - & Inactivo \\
\hline Durango & Estado & Estatal & 2004 & 2009 & - & - & Inactivo \\
\hline \multirow{3}{*}{ Estado de México } & Ecatepec de Morelos & Municipal & 2006 & 2009 & - & - & Inactivo \\
\hline & Tlalnepantla & Municipal & 2014 & 2015 & - & - & Inactivo \\
\hline & Naucalpan & Municipal & 2014 & 2014 & - & - & Inactivo \\
\hline Hidalgo & Mineral de la Reforma & Municipal & 2014 & 2014 & - & - & Inactivo \\
\hline \multirow{16}{*}{ Jalisco } & Acatlán de Juárez & Municipal & 2016 & - & - & - & Activo \\
\hline & Cihuatlán & Municipal & 2016 & - & - & - & Activo \\
\hline & Cuquío & Municipal & 1993 & 2002 & - & - & Inactivo \\
\hline & Guadalajara & Municipal & 2013 & 2013 & 2016 & - & Activo \\
\hline & Juanacatlán & Municipal & 2016 & - & - & - & Activo \\
\hline & La Barca & Municipal & 2013 & 2013 & - & - & Inactivo \\
\hline & Lagos de Moreno & Municipal & 2013 & 2013 & - & - & Inactivo \\
\hline & Tepatitlán de Morelos & Municipal & 2013 & 2013 & - & - & Inactivo \\
\hline & Tlaquepaque & Municipal & 2010 & 2012 & 2016 & - & Activo \\
\hline & Tlajomulco & Municipal & 2011 & - & - & - & Activo \\
\hline & Zapopan & Municipal & 2016 & - & - & - & Activo \\
\hline & Tonalá & Municipal & 2016 & - & - & - & Activo \\
\hline & Zacoalco & Municipal & 2016 & - & - & - & Activo \\
\hline & Zapotlán & Municipal & 2016 & - & - & - & Activo \\
\hline & Puerto Vallarta & Municipal & 2013 & - & - & - & Activo \\
\hline & Etzatlán & Municipal & 2013 & 2014 & - & - & Inactivo \\
\hline \multirow[t]{2}{*}{ Michoacán } & $\begin{array}{l}\text { Con indígenas y zonas } \\
\text { rurales }\end{array}$ & Estatal & 2009 & 2011 & - & - & Inactivo \\
\hline & Numarán & Municipal & 2013 & 2013 & - & - & Inactivo \\
\hline Nayarit & Tepic & Municipal & 1970 & 1972 & 2015 & - & Activo \\
\hline \multirow[t]{2}{*}{ Nuevo León } & $\begin{array}{l}\text { San Pedro Garza } \\
\text { García }\end{array}$ & Municipal & 2003 & 2009 & 2012 & 2015 & Inactivo \\
\hline & Santa Catarina & Municipal & 1997 & 2000 & 2007 & 2011 & Inactivo \\
\hline \multirow{2}{*}{ Puebla } & San Pedro Cholula & Municipal & 2014 & - & - & - & Activo \\
\hline & Puebla & Municipal & 2011 & 2012 & 2015 & - & Activo \\
\hline Quintana Roo & Solidaridad & Municipal & 2009 & 2011 & - & - & Inactivo \\
\hline Sonora & $\begin{array}{l}\text { Por el estado en } \\
\text { municipios }\end{array}$ & Estatal & 2013 & 2013 & - & - & Inactivo \\
\hline
\end{tabular}

Fuente: Elaboración propia.

M. L. García Bátiz, L. Téllez Arana | El presupuesto participativo: un balance de su estudio y evolución en México Perfiles Latinoamericanos, 26(52) | FLACso México | DOI: 10.18504/pl2652-012-2018 


\section{Contexto socioeconómico de las experiencias}

En el mapa 1 se observa el comportamiento de la dispersión en la aplicación de PP por parte de cuatro gobiernos de entidades federativas que representan el $13 \%$ del total de las 32 entidades federativas del país, considerando que este tiene 31 estados y el D. F.; las coberturas son dispares y se comprueba que el D. F. es el único en donde se implementa en todas sus delegaciones, mientras que en los demás estados se limita, por ejemplo, a regiones con población indígena, como en Michoacán, o a grupos selectos, como en Durango y Sonora.

Mapa 1. Presupuestos participativos implementados por gobiernos estatales en México hasta 2016

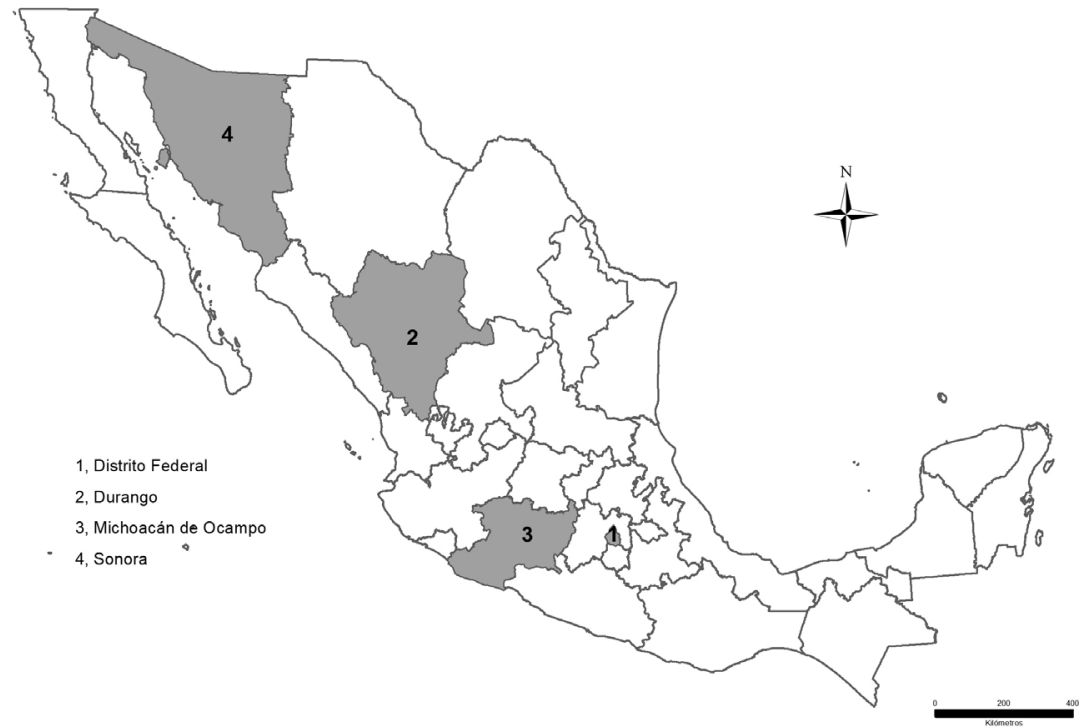

Fuente: Elaboración propia

La población de las entidades resaltadas en el mapa 1 equivale al 16\% del total del país. Sobresale el D. F. por ser mayormente urbano y tener aproximadamente nueve millones de habitantes (INEGI, 2010), esto es, el 8\% del total nacional. Según el Conapo (2010), los grados de marginación de esa población son muy bajo para el D. F., bajo para Sonora, medio para Durango y alto para Michoacán. 
En el mapa 2 se observa cómo la extensión geográfica y numérica de las 33 experiencias en municipios y delegaciones mexicanas es discontinua. Si se recuerda que México cuenta a la fecha con 2440 municipios y 16 delegaciones, o 2456 gobiernos municipales y delegacionales (INAFED, 2015), entonces solo el $1.64 \%$ de ese total han implementado el PP y solo se ha beneficiado al 13\% de la población del país.

Mapa 2. Presupuestos participativos implementados por gobiernos municipales y delegaciones en México hasta 2016

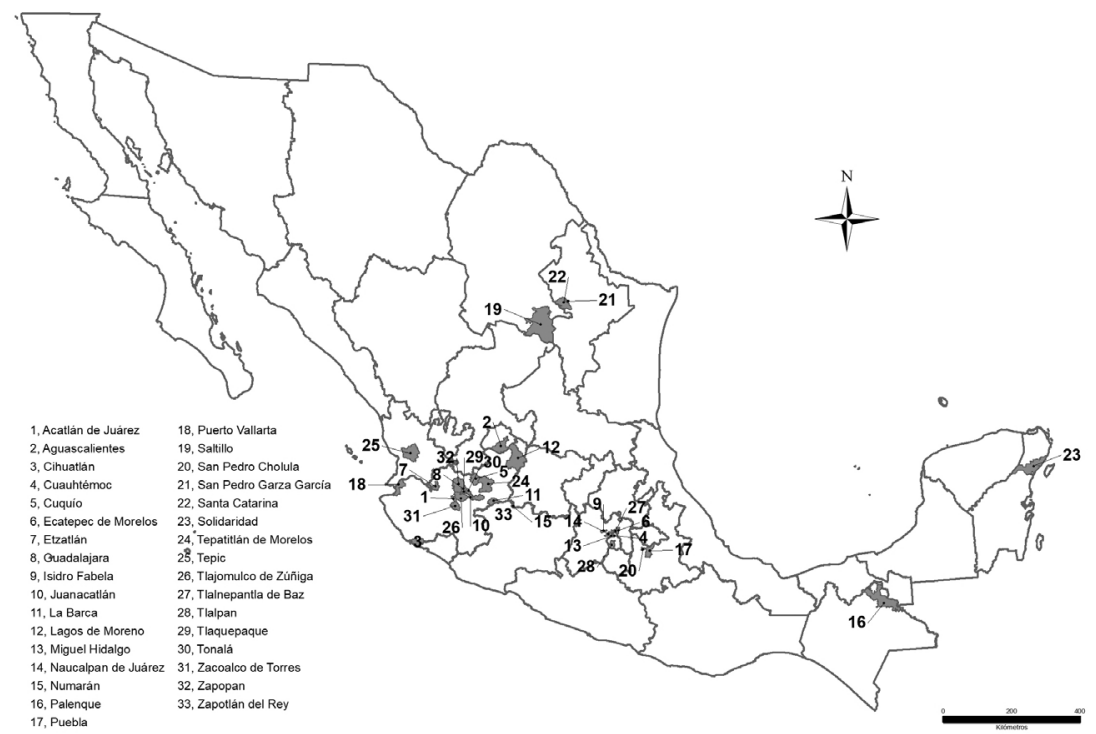

Fuente: Elaboración propia.

Para observar las características de las localidades en las que ha habido experiencia de PP se ha retomado la clasificación de Villalobos (1986. Citado en Sánchez, 2008), quien señala que los municipios son grandes y urbanos si tienen más de cien mil habitantes; serán medianos y semiurbanos, si tienen entre veinticinco y cien mil, y pequeños o rurales con menos de veinticinco mil. Así, el 73\% de los PP ha sido implementado por gobiernos municipales urbanos grandes, $9 \%$ por medianos semiurbanos y $18 \%$ por pequeños rurales. En tanto que al usar los criterios del Conapo (2010), 61\% del total municipal pertenece a zonas metropolitanas. Los grados de marginación de la población de estos municipios asimismo son importantes. Con datos del Conapo (2010) 
se sabe que el $76 \%$ de los PP se han implementado en municipios con muy baja marginación, $15 \%$ en los de baja marginación, $6 \%$ en los de marginación media y $3 \%$ en los de alta marginación.

\section{Condiciones institucionales}

En esta sección se exploran las condiciones institucionales de las experiencias con presupuestos participativos. Para ello se revisan los contextos legales de referencia en el nivel nacional y local, el presupuesto involucrado, el arribo de nuevos gobiernos y el papel de los actores.

México es un país federado con tres órdenes de gobierno que tienen facultades distintas para reglamentar la participación ciudadana. No existe una ley nacional para este aspecto, por lo que cuando se trata de la normatividad del PP esta emana de la instancia estatal o municipal.

La Constitución del país no deja claras las responsabilidades de las entidades federativas sobre la participación ciudadana y solo lo alude para el D. F. en su artículo 122. En la mayoría de las legislaciones y estatutos locales, la participación ciudadana se inserta en términos generales y sin particularizar en el PP. Con todo, en 22 entidades hay leyes de participación ciudadana en las cuales se halló una paulatina institucionalización del PP, el cual está institucionalizado en el D. F. y en cuatro estados más, ${ }^{16}$ el ejercicio del PP sigue activo en el D. F., en Sonora lo estuvo por un año, y de los demás estados no hay registro de su operación.

En 1999, en el artículo 115 de la Constitución mexicana se estableció la obligación de los municipios de asegurar la participación ciudadana y vecinal en la gestión municipal; sin embargo, en las leyes estatales dicha obligación no es homogénea. De igual modo, no todas las experiencias municipales se han recogido en reglamentos, los pocos ejemplos son Tlajomulco de Zúñiga, Guadalajara y Cuquío, donde se ha elaborado un reglamento interno (Arzaluz, 2013: p. 190). En Lagos de Moreno, Tepic y Puebla, se han generado reglas de operación. Mientras que del resto de las entidades no se tuvo datos.

Las responsabilidades institucionales de la implementación del PP en los estados se asocian al área de Planeación, en Michoacán, de Finanzas y Administración Pública, en Durango, y al Ejecutivo, a la Asamblea Legislativa, a los gobiernos delegacionales y el Instituto Electoral local, en el D. F. En los municipios regularmente esas responsabilidades quedan a cargo de las direcciones de participación ciudadana, desarrollo social o de planeación municipal, aunque

16 Colima en 2015, Jalisco en 2016, Michoacán en 2015 y Sonora en 2011. 
en Tonalá esto fue atendido en su momento por el gobierno estatal. En otros municipios de Jalisco las consultas se dieron en coordinación con el Instituto Electoral y el de Participación Ciudadana de Jalisco que facilitó urnas electrónicas y revisó los resultados.

La dimensión financiera de los PP puede comprenderse conociendo la coordinación fiscal entre los tres órdenes de gobierno del país. De acuerdo a Sánchez \& Rosas (2016), el sistema fiscal federal mexicano es dual y en este cada nivel de gobierno cuenta con atribuciones y obligaciones excluyentes lo que comprende que pueden imponer tasas de cobro y gastarlas; así, a decir de Sánchez \& Rosas (2016), los ingresos de los gobiernos estatales son insuficientes pues carecen de algún impuesto relevante, mientras que los municipales tienen como atribución principal cobrar el impuesto a la propiedad si bien sus ingresos dependen fundamentalmente de las participaciones y aportaciones estatal y federal. La tendencia del sistema fiscal mexicano, en suma, es centralizadora y de pocas capacidades locales.

Por la razón mencionada sobre el sistema fiscal federal mexicano, es complicada la identificación de los orígenes y montos del PP para cada gobierno, no obstante, se pueden identificar aproximaciones. En el D. F. se le destina el 3\% del presupuesto anual de cada delegación dividido en partes iguales entre el número de colonias y pueblos originarios. En Michoacán se ha invertido el $0.6 \%$ del presupuesto estatal; en Durango fue creada una bolsa estatal anual con un promedio de medio millón de pesos, y en Sonora fueron 234 millones de pesos, aunque en estos dos estados no se precisa el origen de los recursos.

Para gran parte de los municipios, la partida que se destina al PP corresponde a sus ingresos por el cobro de predial y agua con aportaciones promedio de entre el 10 y el 15\% de lo recaudado por sus haciendas; aunque en Ecatepec de Morelos se combinaron fondos municipales de transferencias, subsidios y recursos propios y en Tonalá el gobierno estatal puso en marcha acciones entre sus dependencias y el municipio para implementar el PP en una colonia. Un pequeño número de municipios ha ligado el $\mathrm{PP}$ a programas y temáticas específicas como seguridad o educación.

Los rubros que se discutieron y que luego recibieron los recursos estatales y municipales estuvieron relacionados casi en su totalidad con obras, servicios públicos, infraestructura y equipamiento para áreas gubernamentales. Un hecho notable es que los periodos para decidir la aplicación de un PP fueron anuales, pero hubo algunos en que esto se extendió a más de un año.

La aparición de las experiencias con PP con frecuencia coincide con el arribo de grupos de filiación política distinta a los de gestiones previas y su duración coincide con su permanencia. Sin afirmar que esto sea decisivo así queda manifiesto el partido político que lidera las gestiones del PP. Para el D. F. y Michoacán 
quedó asociado al PRD, de orientación socialdemócrata. En Durango, al PRI y en Sonora, al pan, ambos tradicionales y conservadores. Para los municipios, el $33 \%$ del total de dichas experiencias se relacionaron con el partido Movimiento Ciudadano y un $18 \%$ al PRD, los dos de orientación socialdemócrata; ${ }^{17}$ otro $27 \%$ al PAN y $21 \%$ al PRI.

Esta investigación localizó experiencias con influencia de más actores en el origen y desarrollo del PP. Así, la institucionalización de este mecanismo en el D. F. se debió a la intervención del Poder Legislativo local por lo que su aplicación al siguiente año se dio en todas las delegaciones de la entidad. Ya se mencionó a Cuquío, donde la comunidad campesina influyó en la gestión del partido entrante, lo que a su vez, impulsó el PP, cuyo diseño fue revisado por organizaciones independientes mediante la participación ciudadana desde varias comunidades. En Tonalá fue implementado por el gobierno estatal. En Ecatepec, su diseño e implementación lo acompañó el organismo social Centro Operacional de Vivienda y Poblamiento, A. C. (Copevi)' el de Tlalnepantla, por la onU-Habitat, con capacitación y revisión del diseño. Fue posible advertir que los gobiernos federal y estatales están ausentes en su promoción o respaldo.

\section{Dinámicas de participación}

Las dinámicas de participación que operan en las experiencias de PP se reconocen analizando los elementos y dimensiones que miden los fines, alcances, intensidades y resultados de la participación (Arnstein, 1969; Brugué y Martí, 2011; Cabannes, 2005; Fung, 2006; Font, Della Porta \& Sintomer, 2014; Gomà y Font, 2001). Así, las dimensiones que dan respuesta a preguntas sobre el potencial, limitaciones y resultados de la participación serían para qué se participa, quiénes participan, cómo participan, hasta dónde participan, en qué asuntos participan y cuáles son las consecuencias de la participación. Para los PP en el país se analizan enseguida las dimensiones de objetivos, intensidad y alcance.

Respecto a los objetivos del PP se ha observado su orientación a la apertura, esto es, si expresan explícitamente la intención de promover y abrir espacios para la participación ciudadana en la decisión de gasto de los recursos públicos locales, así como la de fortalecer los valores democráticos como el capital y el pluralismo social, la igualdad de oportunidades o la rendición de cuentas, y qué tanto las dinámicas de participación contribuyen a su cumplimiento.

17 En Tepic, Nayarit, gobernó el Partido Popular Socialista (pPs). 
La cantidad y orientación de objetivos ${ }^{18}$ de las experiencias de PP son muy distintas y no todas se plantean con claridad. A pesar de esto es posible identificar sus similitudes. En su mayor parte se plantean la apertura e incorporación de la ciudadanía en las decisiones públicas de los recursos públicos, esto es, en las decisiones de gobierno, el empoderamiento ciudadano, la gestión y control del gasto público, la distribución equitativa de los recursos, la generación de capacidades ciudadanas, la creación de capital social y la construcción de nuevas relaciones sociales. De igual modo, integran objetivos orientados a la gestión pública municipal, como el fomento del pago de impuestos para aumentar la contribución local, el conseguir un manejo eficiente y eficaz de los recursos, el modernizar la administración pública y el afirmar la autonomía local (tabla 2).

Tabla 2. Ejemplos de objetivos de presupuestos participativos en México

\begin{tabular}{|c|c|}
\hline Gobierno & bjetivo \\
\hline Cuquío & $\begin{array}{l}\text { "Garantizar a los habitantes del municipio una mayor participación y atención a sus necesidades eco- } \\
\text { nómicas, sociales y culturales mediante la colaboración conjunta entre autoridades y ciudadanía para } \\
\text { lograr el desarrollo integral del municipio." (Rodríguez \& Quintana, 2003: p.158). }\end{array}$ \\
\hline Tlalpan & $\begin{array}{l}\text { Loadyuvar a la construcción de una ciudadanía activa, con derechos y obligaciones, con una visión de } \\
\text { ediano y largo plazo que busque el beneficio de la mayoría. Que sea crítica, propositiva y corresponsable. } \\
\text { avorecer programas que lleven a la consecución de una autonomía real, en la que sean las delegacio- } \\
\text { es las que decidan sobre sus recursos [...] } \\
\text { ortalecer la democracia como práctica en la relación horizontal entre gobierno y sociedad, mejorando } \\
\text { calidad de vida y convivencia social, así como nuevas formas de organización y participación ciuda- } \\
\text { ana." (Delegación Tlalpan \& CESEM, 2003: 62-63. Citado en Delgado, 2006: p. 128). }\end{array}$ \\
\hline Tlajomulco & $\begin{array}{l}\text { "Que los ciudadanos ejerzan el poder de decidir el destino de sus impuestos. } \\
\text { Fortalecer el vínculo entre el gobierno y los ciudadanos al momento de tomar decisiones. } \\
\text { Eficientar [sic] el gasto público, orientándolo a partir de las necesidades planteadas por los ciudada- } \\
\text { nos." (Ayuntamiento de Tlajomulco, 2016). }\end{array}$ \\
\hline & $\begin{array}{l}\text { "Formar capital social con las herramientas necesarias para incidir en las políticas públicas, mediante } \\
\text { la capacitación ciudadana." (IAPEM, 2015). }\end{array}$ \\
\hline D. F. & $\begin{array}{l}\text { "Definir los proyectos específicos en que se aplicarán los recursos de presupuesto participativo corres- } \\
\text { pondiente al ejercicio fiscal inmediato en todas y cada una de las colonias y pueblos originarios en que } \\
\text { se divide el territorio del Distrito Federal." (Ley de Participación Ciudadana del Distrito Federal, 2004). }\end{array}$ \\
\hline Cuauhtémoc & $\begin{array}{l}\text { "Consolidar procesos democráticos, con mayor participación y control de la sociedad sobre los asun- } \\
\text { tos públicos. } \\
\text { Construir una nueva relación y cultura política dando fin a las políticas clientelistas, paternalistas y au- } \\
\text { toritarias." (Luévano, 2002. Citado en Delgado, 2006: p. 126). }\end{array}$ \\
\hline La Barca & $\begin{array}{l}\text { "Dar mayor poder de decisión a la ciudadanía sobre las obras que se realizan en el Municipio." (Ayun- } \\
\text { tamiento de la Barca, 2012). }\end{array}$ \\
\hline $\begin{array}{l}\text { Ecatepec } \\
\text { de Morelos }\end{array}$ & $\begin{array}{l}\text { "Estimular a las comunidades a incrementar la contribución anual del pago de impuestos." (Ayunta- } \\
\text { miento de Ecatepec de Morelos, 2007: 6. Citado en Ramírez, 2011: p. 114). }\end{array}$ \\
\hline Tepic & $\begin{array}{l}\text { "Propiciar una distribución justa, democrática y equitativa de los recursos públicos del Municipio, me- } \\
\text { diante un mecanismo público, objetivo, transparente y auditable, que posibilita a la ciudadanía intervenir } \\
\text { en la solución de los problemas prioritarios de las comunidades, sin exclusiones ni condicionamientos" } \\
\text { (Ayuntamiento de Tepic, 2015). }\end{array}$ \\
\hline
\end{tabular}

Fuente: Elaboración propia con base en Ayuntamiento de Ecatepec de Morelos (2007: 6. Citado en Ramírez, 2011: p. 114), Ayuntamiento de la Barca (2012), Ayuntamiento de Tepic (2015), Ayuntamiento de Tlajomulco (2016), Delegación Tlalpan \& CESEM (2003: 62-63. Citado en Delgado, 2006: p. 128), IAPEM (2015), Ley de Participación Ciudadana del Distrito Federal (2004), Luévano (2002. Citado en Delgado, 2006: p.126), Rodríguez \& Quintana (2003: p.158).

18 Dos estatales: D. F. y Durango. Dieciocho municipales: Tlalpan, Miguel Hidalgo, Cuauhtémoc, Ecatepec de Morelos, Tlalnepantla, Acatlán de Juárez, Cihuatlán, Cuquío, La Barca, Lagos de Moreno, Tlaquepaque, Tlajomulco, Zapopan, Etzatlán, Tepic, San Pedro Garza García, San Pedro Cholula y Puebla. 
No obstante, a pesar de que los PP se proponen grandes objetivos democráticos, en la práctica se observa que su cumplimiento es limitado, lo que se debe a que la intensidad y el alcance de la participación están restringidos o no se relacionan directamente con sus propios objetivos, a excepción de ejemplos como el de Cuquío, Jalisco, o los del D. F. En otros, tienen más peso los objetivos de gestión pública (v. g., el aumento de la recaudación), lo que desalienta el crecimiento de la participación. Así sucede en Tlajomulco de Zúńiga y similares, como se revisa más abajo. ${ }^{19}$

Para revisar la intensidad y el alcance de la participación en la práctica se han considerado varios criterios. La intensidad se ha clasificado de muy baja a alta según favorezca una participación que incluya a la población territorial y temáticamente; busque visibilizar a los sectores tradicionalmente excluidos con acciones afirmativas o de discriminación positiva; combine bases de participación personal, asociativa y mixta, y priorice el diálogo y el debate entre los participantes. El alcance ha sido catalogado de muy bajo a alto de acuerdo al momento de la decisión y gestión de la política en el que se involucre a la sociedad; en cuanto a este punto, se espera que los PP vayan más allá del momento de la priorización y decisión vinculante de opciones para alcanzar niveles en los que las responsabilidades se deleguen a la sociedad para controlar y gestionar los proyectos, y que busquen la inclusión de la ciudadanía en la definición, diseño y evaluación de los procesos y resultados y si se destinan los recursos a una agenda amplia de asuntos locales.

En primer lugar ${ }^{20}$ se presentan experiencias de muy bajo alcance e intensidad, en las que el nivel máximo de participación es la prioridad de la agenda de obras sin implicar decisiones vinculantes ni un diálogo plural, donde las consultas más bien son asambleas informativas o encuestas aplicadas a grupos sociales selectos como empresarios, líderes comunitarios e incluso delegados de gobierno. Con ciertas variaciones, en esta dinámica se encuentran Durango, la delegación Miguel Hidalgo, Mineral de la Reforma y Saltillo, es decir, un 12\% del total de los PP en estudio.

Después se encuentra un grupo de intensidades bajas en el que se han creado consejos ciudadanos únicos de poca inclusión y con medios de representatividad

19 En experiencias semejantes, se fijan objetivos para dotar de poder de decisión a la sociedad; sin embargo, se observa que se promueven a manera de herramienta para aumentar la recaudación municipal, de ahí que solo sea consultada a través de una boleta a la sociedad que paga el impuesto municipal, lo que desarticula el ejercicio de participación en los objetivos fijados.

20 Cuatro estatales: D. F., Durango, Michoacán y Sonora. Veintisiete municipales: Saltillo, Tlalpan, Miguel Hidalgo, Cuauhtémoc, Ecatepec de Morelos, Tlalnepantla, Mineral de la Reforma, Acatlán de Juárez, Cihuatlán, Cuquío, Guadalajara, Juanacatlán, La Barca, Lagos de Moreno, Tlaquepaque, Tlajomulco, Zapopan, Tonalá, Zacoalco, Zapotlán, Puerto Vallarta, Etzatlán, Tepic, San Pedro Garza García, Santa Catarina, San Pedro Cholula y Puebla. 
imprecisos o simples por territorio. El alcance de estos consejos es bajo porque funcionan mediante reuniones esporádicas convocadas por el propio gobierno, y aunque su singularidad reside en que su decisión es vinculante, se enfocan en asuntos de servicios y obra pública barrial sin evidenciar innovaciones en sus procesos, aunque algunos combinan consultas básicas. Son ejemplos, Sonora, Tonalá y La Barca, un 10\% del total.

Luego están las experiencias de intensidad media, pero de bajo alcance. Son los PP focalizados en temáticas o grupos poblacionales específicos, lo que les facilita la intensidad al ubicar en concreto a su población y usar técnicas de diálogo con consultas de peso más decisivo. Su problema es su alcance que se debe a su limitación temática donde los principales resultados son para obra pública e infraestructura. Son ejemplos, Michoacán y la consulta infantil en escuelas de Guadalajara, que suman entre ambos el 6\% del total.

En las experiencias de intensidad muy baja con alcances medios, la decisión es vinculante pero excluyente pues solo consulta anualmente a la ciudadanía que paga impuestos municipales. Son los PP con más auge y entre estos se encuentran los de Tlajomulco de Zúniga, los de otros municipios de Jalisco y el de Cholula. Son 13 casos que significan el 14\% del total, en su mayoría, activos.

Hallamos también experiencias de intensidad intermedia que combinan comités y asambleas territoriales para dar cobertura a toda la población y repartir por igual los beneficios. Son experiencias que distinguen poco entre los sectores más desfavorecidos y presentan mecanismos deliberativos poco articulados. $\mathrm{Su}$ alcance es medio-bajo dado que se centran en decisiones vinculantes de obra y servicios públicos con una agenda elaborada por los comités que luego ponen a consulta entre la ciudadanía, pero rara vez llegan a niveles de cogestión y evaluación. Con variaciones, aquí se inscriben el D. F. y las delegaciones Cuauhtémoc y Tlalpan, además de Ecatepec de Morelos, Tlalnepantla, Tepic, San Pedro Garza, Puebla y Santa Catarina, en resumen, $28 \%$ del total.

Finalmente, el PP de más intensidad y alcance participativo, es el de Cuquío. La intensidad resultó alta por la combinación de comités territoriales y temáticos que asegura una inclusión amplia de la población dando prioridad a sectores desatendidos como las comunidades campesinas y otros. Asimismo, porque los consejos comunitarios se eligieron en asambleas públicas y tuvieron un carácter autónomo y representativo de sus comunidades; los cuales, reunidos formaban consejos zonales integrados al comité permanente del CODEMUC, tenían el control y vigilancia de los procesos y en sus constantes reuniones y asambleas se deliberaba para evaluar la viabilidad, priorizar y decidir las acciones centradas en servicios y obras públicas, con procesos afinados continuamente.

En la gráfica 2 se clasifican todas estas experiencias. Se han colocado los nombres de las prácticas representativas según las dimensiones analizadas. 


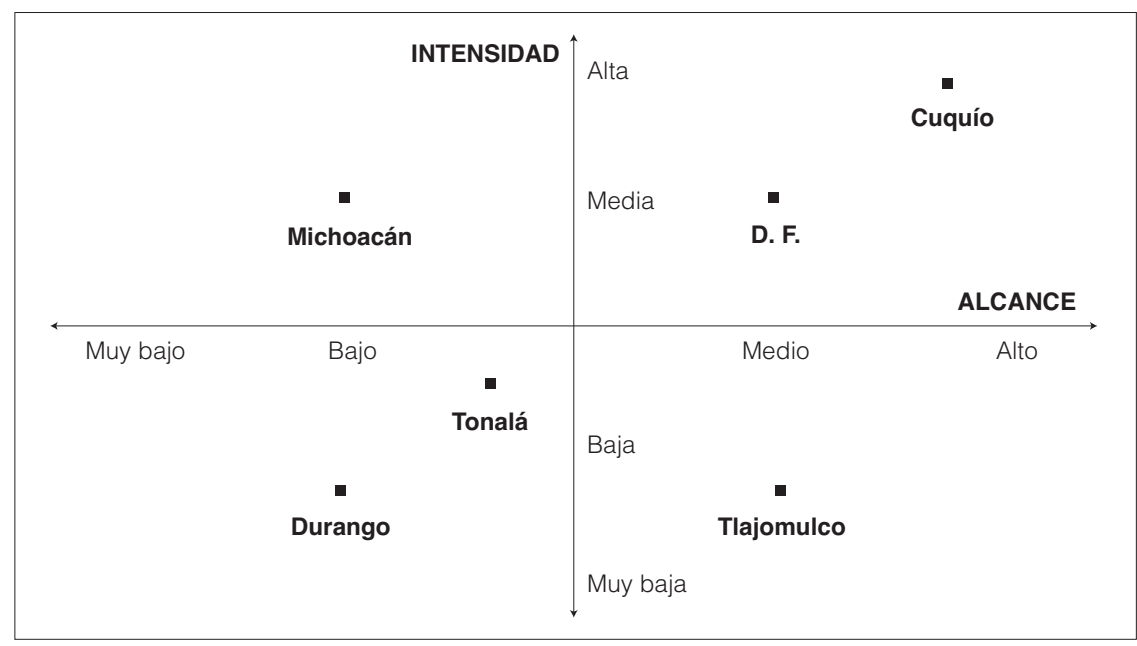

Fuente: Elaboración propia.

\section{Reflexiones finales}

El PP es un mecanismo de participación ciudadana usado cada vez más en sociedades democráticas contemporáneas para profundizar los valores de una democracia participativa, en la cual se ubica a México. En este país es creciente el interés de gobiernos locales, comunidades académicas y sociedad por el PP a fin de utilizarlo y analizarlo como práctica que incorpora a la ciudadanía en las decisiones de los recursos públicos locales. Pero su desarrollo propone retos importantes.

La investigación internacional sobre los PP ha prestado poca atención a las experiencias mexicanas y los estudios locales han divulgado contados casos que no consiguen la sistematización ni analizan los diseños, resultados y explicaciones de dicho mecanismo. Por esto el primer reto de la agenda de investigación sería pasar de la descripción al análisis y la explicación, pero desde una visión más crítica de la democracia, además de que se requiere de los estudios comparados para visibilizar los problemas contextuales, y de ampliar las fuentes de información sobre las experiencias en México, que podrían ser formales o redes internas que promuevan el estudio y extensión del PP.

Por lo anterior este artículo se propuso evaluar el desarrollo del PP en México, identificando, en primera instancia, que hay una extensión territorial y temporal de este tipo de experiencias en crecimiento, sin obstar una tendencia lenta, inconstante y pasajera. 
En relación a los factores analizados, sobre el contexto sociodemográfico se encontró que la aparición y estabilidad de las experiencias del PP están fuertemente ligadas a contextos sobre todo urbanos y localizados en las zonas centro y occidente que son las regiones de mayor avance económico; para construir una democracia más sólida, el segundo reto del PP sería desarrollarlo en localidades y regiones que históricamente han presentado atraso y desigualdades sociales. Esto incentivaría los objetivos democráticos y de gobierno que se formulan con esa herramienta y los consolidaría donde ya se implementan.

De las condiciones institucionales es claro que poco favorecen la aparición y estabilidad del PP en México. En lo legal se evidencia una densidad institucional baja en los tres órdenes de gobierno para respaldarlo y promoverlo; en tanto que el marco fiscal muestra una fuerte centralización que obstaculiza la autonomía de los gobiernos para ampliar la oferta del PP. De igual modo, con el diseño electoral sin reelección en los gobiernos locales, vigente al momento de la investigación, el PP queda sujeto a los vaivenes electorales. Un panorama poco alentador que se subraya con el escaso interés que muestran otros actores sociales para involucrarse, promover y defender a dicho mecanismo.

Con este resumen es posible derivar como hipótesis de trabajo que la aparición y permanencia de los presupuestos participativos en México se relacionan con las condiciones institucionales prevalecientes en el país donde existe una baja densidad institucional participativa, una centralización en el manejo de los recursos financieros, un sistema electoral que da incentivos para su aparición pero ninguno para su continuidad, y una notable ausencia de sectores sociales interesados por promoverlos. Por ello, el tercer reto sería profundizar el conocimiento que verifique esta proposición y mejorar las condiciones institucionales para los gobiernos subnacionales y municipales que propicien la innovación local con participación ciudadana.

Por último, acerca de las dinámicas de participación que se ponen en práctica, el hallazgo ha sido constatar que la mayoría de las experiencias cuentan con grandes objetivos democráticos pero con procesos de participación que contribuyen poco para cumplirlos; y que predominan los PP con dinámicas de alcances e intensidades muy bajas, los cuales dan preferencia a la consulta, no procuran ser representativos, ni inclusivos ni redistributivos, no trascienden la decisión y no muestran intensiones de aprendizaje.

De lo anterior se deriva una segunda hipótesis de trabajo. El diseño de los presupuestos participativos de México depende de las condiciones institucionales dado que los adoptan los grupos políticos entrantes a los gobiernos locales y que actúan en marcos institucionales de débil oferta de participación y los recrean como una política para producir imágenes de contraste con los grupos políticos salientes. Es una herramienta con la que generan relaciones flexibles con secto- 
res selectos de la población y palian problemas financieros locales, lo que refleja su desinterés por modificar las estructuras políticas preestablecidas y por crear políticas abiertas al diálogo e inclusivas de sectores de población con menos oportunidades de desarrollo.

El quinto reto de las experiencias en México no es que simplemente aparezcan y permanezcan, sino que se desarrollen o avancen a diseños de más alcance e intensidad que contribuyan realmente a los objetivos amplios para la construcción de la democracia local que se proponen.

En síntesis, los presupuestos participativos en México presentan avances cuantitativos pausados e inconsistentes con retrasos cualitativos significativos; las condiciones institucionales son un gran factor a superar para su aparición, permanencia y dinámica participativa, pero las experiencias demuestran que pueden ser menos importantes cuando la sociedad se involucra activamente y ese es otro reto difícil de superar en el contexto mexicano.

\section{Referencias}

Arellano, D. (2006). Los dilemas de la gestión local y las organizaciones comunitarias en México. México: CIDE.

Arnstein, S. R. (1969). A ladder of citizen participation. Journal of the American Planning Association, 35(4), 216-224.

Avritzer, L. (2004). Presupuestos participativos: distribución, eficiencia y cultura democrática. La Era Urbana, (Edición especial), 10-12.

Ayuntamiento de Ecatepec de Morelos. (2007). Manual de Operación del Proceso del Presupuesto Participativo 2008. Ecatepec de Morelos.

Ayuntamiento de la Barca. (2012). Programa Operativo Anual. Recuperado en marzo de 2016, de http://www.labarca.gob.mx/info8_15/art_8/frac_4/2/2012/poa_desarrollosocial.pdf

Ayuntamiento de Tepic. (2015). Bases para el presupuesto participativo de Tepic, Nayarit, 2015. Recuperado en marzo de 2016, de http://tepic.gob.mx/archivos/transparencia/xii/III/26_ ACTA_DEL_31_DE_MARZO_DEL_2015_SESION_EXTRAOR.PDF

Ayuntamiento de Tlajomulco. (2016). ¿Qué es el presupuesto participativo?. Recuperado en marzo de 2016, de https://presupuestoparticipativo.mx/

Baiochi, G. (2001). Activism and Politics: The Porto Alegre Experiment and Deliberative Democratic Theory. Politics and Society, 29(1), 43-72. 
Bautista, J. (2011). Retos y agenda para la implementación de presupuestos participativos en la Zona Metropolitana de Guadalajara. Presentado en el Foro Presupuestos Participativos: la participación ciudadana en el gasto público, México.

Bazdresch, M. (2001). Consejo Democrático Municipal: Innovación en Cuquío. En Cabrero, E. (Coord.). Innovación en gobiernos locales: un panorama de experiencias municipales en México (pp. 455-474). México: CIDE.

Bey, M. (2007). Alternancia política y cambios en el escenario político local: el caso de Cuquío. Espiral, XIII(38), 169-195.

Bolos, S. (2003). Organizaciones sociales y gobiernos municipales: construcción de nuevas formas de participación. México: Universidad Iberoamericana.

Briseño, C. A. (2011). Presupuesto participativo. Herramienta para la democracia. México: Congreso del Estado de Jalisco/Instituto Electoral y de Participación Ciudadana de Jalisco.

Brugué, Q. \& Martí, S. (2011). Participación y democracia en el ámbito local. En Briseño, C. A. (Coord.). Presupuesto participativo. Herramienta para la democracia. México: Congreso del Estado de Jalisco/Instituto Electoral y de Participación Ciudadana de Jalisco.

Buenrostro, I. (2004). Ciudadanía y presupuesto participativo: Anotaciones al caso de Porto Alegre como práctica ciudadana. Araucaria, 5(12), 67-82.

Cabannes, Y. (2005). Documento base presupuesto participativo y finanzas locales. Segunda versión ampliada. Porto Alegre, Brasil.

Cabannes, Y. (2004). Participatory budgeting: A significant contribution to participatory democracy. Environment and Urbanization, 16(1), 27-46.

Canto, M. (2010). Participación ciudadana en las políticas públicas. México: Siglo XXI.

Conapo (2010). Indice de marginación por entidad federativa y municipio. México: Conapo.

Delegación Tlalpan \& cesem. (2003). La Experiencia de la Delegación Tlalpan. Poder Ciudadano. Juntos en movimiento. Gobierno y Sociedad. México: Delegación Tlalpan/CESEM.

Delgado, L. C. (2006). Modelo para la gestión de presupuestos participativos en las delegaciones del Distrito Federal. Tesis de Maestría en Proyectos para el Desarrollo Urbano. Universidad Iberoamericana. México.

Díaz, A. \& Ortiz, Á. (2011). Participación ciudadana y gestión pública en los municipios mexicanos: un proceso estancado. En Cabrero, E. \& Arellano, D. (Coords.). Los gobiernos muni- 
cipales a debate: un análisis de la institución municipal a través de la Encuesta INEGI 2009 (pp. 361-432). México: CIDE.

Font, J., Della Porta, D. \& Sintomer, Y. (2014). Participatory Democracy in Southern Europe. Londres: Rowman and Littlefield.

Fung, A. (2006). Varieties of participation in complex governance. Public Administration Review, (66, Special Issue), 66-75.

INEGI (2010). Censo de Población y Vivienda 2010. México: INEGI.

INEgI (2009). Encuesta Nacional de Gobierno, Seguridad Pública y Justicia Municipal. México: INEGI.

INEgi/Indesol. (2000). Encuesta Nacional sobre Desarrollo Institucional Municipal. México: INEGI/INDESOL.

InEgi/Indesol. (1995). Encuesta Nacional sobre Desarrollo Institucional Municipal. México: INEGI/INDESOL.

García, M. (2014). El presupuesto participativo en la Ciudad de México: Un análisis sobre la consecución de los objetivos de profundización democrática y justicia social. Tesina de Maestría en Administración y Políticas Públicas. cide. México.

García, M. L. (2009). Gobernabilidad local: la incorporación de los ciudadanos en la gestión municipal metropolitana. En Mazurek, H. (Ed.). Gobernabilidad y gobernanza de los territorios en América Latina (pp. 455-473). Lima, Perú: Instituto Francés de Estudios Andinos.

García, M. L. (2006). Planeación participativa: la experiencia de la política ambiental en México. México: Plaza y Valdés.

Goldfrank, B. (2012). The World Bank and the globalization of participatory budgeting. Journal of Public Deliberation, 8(2), 7.

Goldfrank, B. (2006). Los procesos de "presupuesto participativo" en América Latina: éxito, fracaso y cambio. Revista de Ciencia Politica, 26(2), 3-28.

Gomà, R. \& Font, J. (2001). La democracia local: un mapa de experiencias participativas. En Font, J. (Ed.). Ciudadanos y decisiones públicas. Barcelona: Ariel.

Gomes, M. A. (2011). Presupuestos participativos. Panorama de Brasil. En Alberto, C. Briseño (Coord.). Presupuesto participativo. Herramienta para la democracia (pp. 39-46). México: Congreso del Estado de Jalisco/Instituto Electoral y de Participación Ciudadana de Jalisco. 
Gómez, E. (2007). El presupuesto participativo entre democracia, pobreza y desarrollo. Investigación \& Desarrollo, 15(1), 56-77.

Gurza, A. \& Isunza, E. (2010). Precisiones conceptuales para el debate contemporáneo sobre la innovación democrática: participación, controles sociales y representación. En Vera, E. \& Gurza, A. (Coords.). La innovación democrática en América Latina. Tramas y nudos de la representación, la participación y el control social (pp. 19-80). México: ciesas/Universidad Veracruzana.

Hernández, E. \& Velasco, G. (2012). Nuevas políticas públicas: el presupuesto participativo. El caso de los municipios de la Zona Metropolitana de Guadalajara. En Valdez, A., Borrayo, C. L. \& De León, A. (Coords.). Nueva agenda pública para Jalisco (pp. 267-298). México: Universidad de Guadalajara/Prometeo Ediciones.

Hevia, F. \& Isunza, E. (2010). La perspectiva de interfaz aplicada a las relaciones sociedad civilEstado en México. En Olvera, A. (Coord.). La democratización frustrada. Limitaciones institucionales y colonización politica de las instituciones garantes de derechos y de participación ciudadana en México (pp. 59-127). México: CIESAS/Universidad Veracruzana.

IAPEM. (2015). Premio IAPEM a la Gestión Municipal. Recuperado en marzo de 2016, de https:// politicasgobiapem.wordpress.com/2015/11/06/experienciaexitosatlalnepantla-presupuestos-participativos/

INAFED. (2015). Enciclopedia de los municipios y delegaciones de México. México: INAFED.

Ley de Participación Ciudadana del Distrito Federal. (2004). Publicada en la Gaceta Oficial del Distrito Federal el 17 de mayo de 2004. (Versión electrónica). Última reforma publicada en la Gaceta Oficial de la Ciudad de México el 17 de noviembre de 2016.

Luévano, Alejandro (2002). Desafio para uma Gestao Local Democrática. O Orcamento Participativo na Cidade do México. Os Primeiros Passos, Alcances, Limites e Desafios. Presentado en el II Encuentro Internacional de Presupuesto Participativo. 19, 20 y 21 de agosto de 2002, Lima, Perú.

Marquina, M. de L. \& Alonzo, M. (2012). Organización ciudadana para la gestión de los presupuestos participativos en el Distrito Federal. Presentado en el Tercer Congreso Internacional Gobierno, Gestión y Profesionalización en el ámbito local ante los grandes restos de nuestro tiempo. 22, 23 y 24 de octubre de 2012, Universidad de Sonora, México.

Merino, M. (Coord.). (1994). En busca de la democracia municipal. Participación ciudadana en el gobierno local mexicano. México: El Colegio de México. 
Montecinos, E. (2014). Diseño institucional y participación ciudadana en los presupuestos participativos. Politica y Gobierno, XXI(2), 351-378.

Montecinos, E. (2011). Democracia participativa y presupuesto participativo en Chile: ¿Complemento o subordinación a las instituciones representativas locales? Revista de Ciencia Política, 31(1), 63-89.

Montecinos, E. (2005). Instituciones políticas y participación social en el espacio local. Revista Austral de Ciencias Sociales, (9), 3-14.

Navarro, Z. (1998). Democracia y control social de fondos públicos: El caso del presupuesto participativo de Porto Alegre. Recuperado en mayo de 2016, de http://unpan1.un.org/intradoc/ groups/public/documents/clad/unpan000172.pdf

Nylen, W. (2003). Participatory Democracy versus Elitist Democracy: Lessons from Brazil. Nueva York: Palgrave Macmillan.

Oliveira, O. P. (2011). Las dinámicas de transferencia del presupuesto participativo: una mirada hacia la acción de las élites. En Welp, Y. \& Whitehead, L. (Coords.). Caleidoscopio de la innovación democrática en América Latina (pp.119-144). México: Flacso México.

Olvera, A. (2010). Introducción. Instituciones garantes de derechos y espacios de participación ciudadana en una transición frustrada. En Olvera, A. (Coord.). La democratización frustrada: limitaciones institucionales y colonización politica de las instituciones garantes de derechos y de participación ciudadana en México (pp. 13-57). México: CIESAs/Universidad Veracruzana.

Olvera, A. (2009). La participación ciudadana y sus retos en México. Un breve estudio del desarrollo de la cultura y de las instituciones participativas y diagnóstico de su problemática actual, con propuestas para hacer funcionales las instancias de participación democrática. México. Recuperado de http://www.gobernacion.gob.mx/work/models/SEGOB/Resource/946/5/images/b) Olvera_Entregable_2.pdf

Ramírez, G. (2011). El presupuesto participativo, experiencia del municipio de Ecatepec de Morelos. En Briseño, C. A. (Coord.). Presupuesto participativo. Herramienta para la democracia (pp. 107-124). México: Congreso del Estado de Jalisco/Instituto Electoral y de Participación Ciudadana de Jalisco.

Rodríguez, C. \& Quintana, R. D. (2003). Las incongruencias de la participación ciudadana y la descentralización en municipios rurales: el caso de Cuquio, Jalisco. Recuperado en mayo de 2016, de http://www.flacsoandes.edu.ec/biblio/catalog/resGet.php?resId=25225

Rodríguez R., Annett K. \& Arango X. (2013). Presupuestos Participativos como herramienta de consolidación democrática. Presentado en el Décimo Congreso Nacional y Tercer Congreso 
Internacional del colparmex. 7 y 8 de octubre de 2013, Universidad Autónoma de Chiapas, México.

Sánchez, A. (2008). Cambio institucional y desempeño de los gobiernos municipales en México. México: Academia Jalisciense de Ciencias/Plaza y Valdés.

Sánchez, A. \& Rosas, J. (2016). Retos de los gobiernos municipales para financiar el desarrollo Metropolitano en Jalisco. Ponencia presentada en el II Coloquio de Invierno de la Red de Políticas Públicas Jalisco: Problemas y capacidades de respuesta. 23, 24 y 25 de febrero de 2016, Guadalajara, Jalisco, México.

Schneider, C. (2007). La participación ciudadana en los gobiernos locales: contexto político y cultura politica. Un análisis comparado de Buenos Aires y Barcelona. Tesis del Doctorado en Teoría Política y Social. Universidad Pompeu Fabra, Barcelona.

Schneider, C. \& Welp, Y. (2011). ¿Transformación democrática o control político? Análisis comparado de la participación ciudadana institucional en América del Sur. Íconos. Revista de Ciencias Sociales, (40), 21-39.

Sintomer, Y. (2005). Los presupuestos participativos en Europa: retos y desafios. Ix Congreso Internacional del CLAD sobre la Reforma del Estado y de la Administración Pública. 2 al 5 de noviembre de 2005. Madrid. Espańa.

Sintomer, Y., Herzberg, C. \& Allegretti, G. (2010). Learning from the South: Participatory Budgeting Worldwide-an Initation to Global Cooperation: Study. Alemania: Service Agency Communities in One World.

Sousa, S. (2004). Democracia y participación. El ejemplo del presupuesto participativo de Porto Alegre. Ecuador: El Viejo Topo.

Tellez, L. \& García, M. L. (2016). Análisis de las determinantes institucionales para el diseño de presupuestos participativos en México. En Ángel, H. \& García, N. (Coords.). Análisis de Politicas públicas regionales: Acercamientos y aportaciones (pp. 110-136). México: Universidad de Guadalajara.

Wampler, B. (2004). Instituçôes, associações e interesses no orçamento participativo de São Paulo. En Avritzer, L. (Coord.). Associativismo em São Paulo. Sao Paulo: Edusp.

Wampler, B., Lubambo, C., Coelho, D. \& Melo, M. (2006). ¿Expandiendo la responsabilidad en la gestión por medio de instituciones participativas? Activistas y reformistas en los municipios brasileños. En Clacso (Ed.). Diseño institucional y participación política. Experiencias en el Brasil contemporáneo (pp. 39-71). Buenos Aires: CLACso. 
Welp, Y. (2016). La participación ciudadana como compromiso democrático. Revista Mexicana de Derecho Electoral, (10), 97-121.

Ziccardi, A. (2004). Espacios e instrumentos de participación ciudadana para las políticas sociales del ámbito local. En Ziccardi, A. (Coord.). Participación ciudadana y politicas sociales en el ámbito local (pp. 245-272). México: IIS-UNAM/COMECSO/INDESOL.

Ziccardi, A. (1998). Gobernabilidad y participación ciudadana en la Ciudad Capital. México: IIS-UNAM.

Ziccardi, A. \& Solano, S. A. (1995). La tarea de gobernar: las ciudades y la gobernabilidad. En Ziccardi, A. (Coord.). La tarea de gobernar: gobiernos locales y demandas ciudadanas (pp. 13-37). México: IIS-UNAM. 